\title{
SEARCHING FOR FAILED SUPERNOVAE WITH ASTROMETRIC BINARIES
}

\author{
ANDrew Gould AND SAMIr SALIM \\ Department of Astronomy, Ohio State University, 140 West 18th Avenue, Columbus, OH 43210; \\ gould@astronomy.ohio-state.edu, samir@astronomy.ohio-state.edu \\ Received 2001 November 27; accepted 2002 February 27
}

\begin{abstract}
Stars in the mass range $8 M_{\odot} \lesssim M \lesssim 30 M_{\odot}$ are thought to end their lives as luminous supernovae that leave behind a neutron star. However, if a substantial fraction of these stars instead ended as black hole remnants, without producing a supernova (a "failed" supernova), how would one know? We show that, under plausible assumptions, the Hipparcos catalog should contain $\sim 30 f_{\text {fail }}$ astrometric binaries with black hole companions, where $f_{\text {fail }}$ is the fraction of supernovae that fail. Since no black hole astrometric binaries are found in Hipparcos, one might like to conclude that such failed supernovae are very rare. However, the most important assumption required for this argument, the initial companion mass function (ICMF) of G stars (the majority of Hipparcos stars) in the high-mass companion regime, is without any observational basis. We show how the ICMF of G stars can be measured using future space-based astrometric missions, thereby permitting an accurate measurement of the rate of supernovae that fail.
\end{abstract}

Subject headings: astrometry — binaries: general — stars: neutron — supernovae: general

On-line material: color figures

\section{INTRODUCTION}

Massive stars $\left(M \gtrsim 8 M_{\odot}\right)$ end their lives as supernovae $(\mathrm{SNe})$, leaving behind black hole $(\mathrm{BH})$ or neutron star (NS) remnants depending on whether they are more or less massive than a cutoff mass, $M_{\text {cut }} \sim 30 M_{\odot}$. So says the standard lore, but what is the observational evidence?

Certainly there exist NSs and BHs, and since pulsars are frequently found near the centers of SN remnants, there can be little doubt about their origin. But do the majority of massive stars $8 \lesssim M / M_{\odot} \lesssim 30$ really die gloriously in luminous $\mathrm{SNe}$ that give birth to a NS? Or do most of them fizzle out in failed SNe that collapse in on themselves, leaving behind a $\mathrm{BH}$ ?

Failed $\mathrm{SNe}$ have been invoked by theorists for a number of reasons, mostly because of the difficulties of producing an explosion in analytical models and simulations (for a detailed review see Woosley \& Weaver 1986). The failures in the earliest models in the 1960s were mostly ascribed to the models' inability to transfer the energy of the core collapse into decoupling the mantle from the envelope. Improvements in the neutrino transport model and new estimates of neutrino cross sections still failed to produce explosions in the 1970s. Owing to improved nuclear rates and more sophisticated models, successful explosions were finally produced in the 1980s, however, only for low-mass iron cores $\left(M \lesssim 1.3 M_{\odot}\right)$, corresponding to progenitor masses of $M \lesssim 11 M_{\odot}$. This problem was somewhat alleviated by the introduction of a delayed neutrino energy transport, which supplies the energy to the lower parts of envelope and so helps the shock propagate outward. The success of this mechanism remains inconclusive. One should keep in mind that the researchers were driven to make explosions happen and not to demonstrate that they do not happen. Mikaelian (1978) early on suggested that some SNe really "fail," arguing that only stars that spin slowly can produce a SN, while the fast spinning stars just collapse into a BH. Others, however, have dismissed rotation as a significant factor. Failed $\mathrm{SNe}$ (of Type Ib) were suggested by Woosley (1993) to rep- resent the main mechanism for producing cosmological $\gamma$-ray bursts (GRBs). In this conjecture, for which the simulations have already produced substantial support, the collapsing core fails to produce an explosion, but instead an accretion disk forms around the core, which draws in mass from the mantle at the rate of $\sim 0.5 M_{\odot} \mathrm{s}^{-1}$, and then quickly collapses into a BH. Woosley (1993) suggests that in stars that have previously lost their H-envelope (Wolf-Rayet stars) the accretion could be accompanied by an energetic burst of gamma rays in the form of polar jets. This model is the basis for the currently favored "hypernova" scenario, first proposed by Paczyński (1998), which in addition requires the presence of very strong $(\sim 0.1 \mathrm{~T})$ magnetic fields. The theoretical predictions of the frequency of $\mathrm{BH}$ formation relative to NS formation are extremely insecure. These estimates rely on a number of uncertain parameters such as the progenitor cutoff mass $\left(M_{\text {cut }}\right)$, progenitor mass function, maximum possible NS mass, equation of state of nuclear matter, etc. As a result, theoretical estimates of the $\mathrm{BH} / \mathrm{NS}$ ratio range from $\simeq 0$ to $\simeq 3$ (Beacom, Boyd, \& Mezzacappa 2001).

There are remarkably few observational probes of failed $\mathrm{SNe}$. The expected rate of core-collapse $\mathrm{SNe}$ derived from the luminosity function (LF) of massive stars predicts many times fewer SNe than are historically observed in our Galaxy (van den Bergh \& Tammann 1991), most likely indicating poor knowledge of the massive star LF. Therefore, it is not possible to infer from this whether failed SNe exist. Ultimately, with sufficiently sensitive neutrino detectors, one could directly detect many extragalactic $\mathrm{SNe}$ and identify the failed ones among them from the lack of a (or very subluminous) optical counterpart. Furthermore, if the neutrino flux is strong enough, its time evolution enables direct characterization of a remnant object. Namely, the neutrino signal from a forming BH should be cut off abruptly as the event horizon forms (Burrows 1988; Beacom, Boyd, \& Mezzacappa 2000). However, with current detectors, these scenarios can be probed only for Galactic $\mathrm{SNe}$, which are not frequent enough to determine the rate, although they could 
demonstrate that failed SNe exist. Microlensing observations with the Space Interferometry Mission (SIM) can directly measure the masses of isolated $\mathrm{BHs}$ and NSs in Galactic bulge fields (Gould \& Salim 1999; Gould 2000). One could then compare the ratio of NS/BH detections to what would be expected based on, say, a Scalo (1986) mass function. The interpretation would be somewhat complicated by the fact that many NSs receive a large kick at birth, which could remove a large fraction of them from the bulge. Nevertheless, given a sufficiently large sample, and with the information on the kick-velocity distribution gleaned from the NS transverse velocities (which come out of the same microlensing observations), it should be possible to reconstruct the remnant ratio. Finally, one could examine the ratio of NS/BH X-ray binaries, where these remnant objects are routinely found. However, since these are interacting systems with complex evolutionary histories, it is difficult to make inferences about the ratio of total populations, and therefore the production mechanisms, from these very special objects.

Here we propose another probe of failed $\mathrm{SNe}$ : $\mathrm{BHs}$ in astrometric binaries. In astrometric binaries the components are too close to be resolved, and only the motion of the photocenter is observed. If one component is invisible (BH or NS), only the motion of the visible component around the barycenter will be detected. This experiment would be sensitive to detecting failed $\mathrm{SNe}$ that did not undergo some process that would disrupt the system. MacFadyen \& Woosley (1999) predict that the formation of a collapsar might always be accompanied by a hypernova explosion, however, whether this necessarily precludes a $\mathrm{BH}$ from remaining in the binary system is uncertain.

In $\S 2$ we discuss the sensitivity of astrometric surveys to dark companions, focusing specifically on Hipparcos. Note that only space-based surveys probe enough stars to allow an effective search for rare objects. We show that Hipparcos is sensitive to $\mathrm{BH}$ companions of the great majority of stars in its catalog. No BH binaries are found, which potentially places strong limits on the number of failed $\mathrm{SNe}$. However, since the initial companion mass function (ICMF) of Hipparcos stars is unknown, there is a serious loophole in this argument: one does not know whether the absence of $\mathrm{BH}$ astrometric binaries reflects the absence of failed $\mathrm{SNe}$ or the absence of progenitors in binaries. In $\S 3$ we show that astrometric binary searches by future space-based astrometry missions can close this loophole.

\section{ASTROMETRIC DETECTION OF DARK COMPANIONS}

Consider a binary whose components have masses and luminosities in the band of astrometric observations $(M, L)$ and $(m, l)$, respectively. From Kepler's third law, the semimajor axis, $a$, is related to the period, $P$, by $\left[(m+M) / M_{\odot}\right](P / \mathrm{yr})^{2}=(a / \mathrm{AU})^{3}$. If the motion of the photocenter is fit to a Keplerian orbit, the angular semimajor axis of the photocenter orbit, $\alpha$ (measured in the orbital plane), will then be related to the other parameters by

$$
\frac{m^{3}}{M_{\odot}(m+M)^{2}}\left(\frac{L}{L+l}\right)^{3}=\left(\frac{P}{\mathrm{yr}}\right)^{-2}\left(\frac{D \alpha}{\mathrm{AU}}\right)^{3},
$$

where $D$ is the distance to the system. The quantities on the right-hand side of this equation can all be measured astro- metrically. We will assume that $M$, the mass of the more luminous component, can be estimated photometrically or spectroscopically. And we will focus on the case in which the companion is known to be dark (or at least extremely dim compared to the primary), $l \ll L$. Under these assumptions, it is straightforward to determine $m$, the mass of the dark companion, from the astrometric observations.

In general, $\alpha$ can be measured with approximately the same precision as the parallax, $\pi$. Of course this does not hold exactly. Even for circular binary orbits, the inclination of the orbit will not match exactly the ecliptic latitude (i.e., the inclination of the parallactic circle), so there will be either more or less information about the binary orbit than about the reflex motion of the Earth's orbit (parallax). Moreover, for certain binary orbits, notably edge-on highly eccentric orbits that "point" in our direction, the errors in $\alpha$ will be much larger than the parallax errors because the binary will show almost no astrometric motion. And, of course, binaries with periods of $\sim 1 \mathrm{yr}$ are especially difficult to detect because of parallax aliasing. Nevertheless, from the standpoint of making an estimate of the errors for a random ensemble of binaries, setting $\sigma_{\alpha} \sim \sigma_{\pi}$ is a good approximation. This is confirmed by Figure 1, where we plot $\sigma_{\alpha} / \sigma_{\pi}$ for astrometric binaries with orbital solutions (i.e., binaries of type "O") in the Hipparcos catalog (ESA 1997, Vol. 10). While these fits made use of some auxiliary ground-based spectroscopic information (mainly to establish the period), or constrained orbits to be circular, this should not have a major impact on the errors in $\alpha$ for periods $P \lesssim 3.3 \mathrm{yr}$, the duration of the mission. Although the figure shows some scatter, the two errors are of the same order, with $\left\langle\log \left(\sigma_{\alpha} / \sigma_{\pi}\right)\right\rangle=\log (1.22)$.

Figure 2 shows the sensitivity ( $5 \sigma$ detection) of Hipparcos to dark companions as a function of stellar type, i.e., the number of Hipparcos stars that can be probed for companions of a given mass. These types were assigned based on

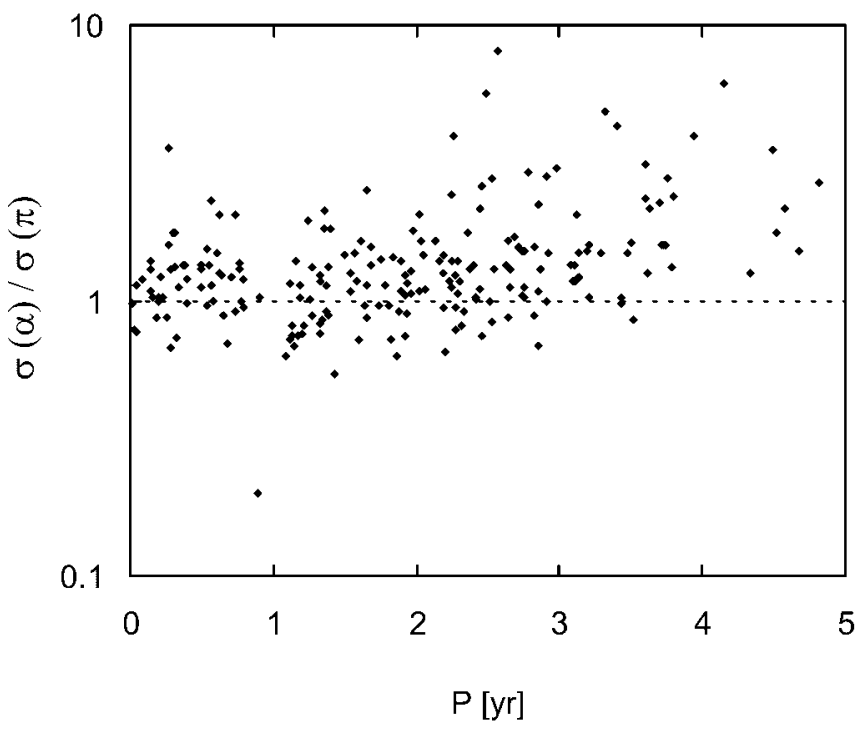

FIG. 1.-Ratio of the error in the photocentric semimajor axis $\sigma_{\alpha}$ to the parallax error $\sigma_{\pi}$ as a function of period $P$ for astrometric binaries with orbital solutions from the Hipparcos catalog. Plot shows 210 systems (four lie outside of the $y$-axis range). As expected from general arguments, the ratio is typically unity. Note that the Hipparcos mission lasted for 3.3 years. 


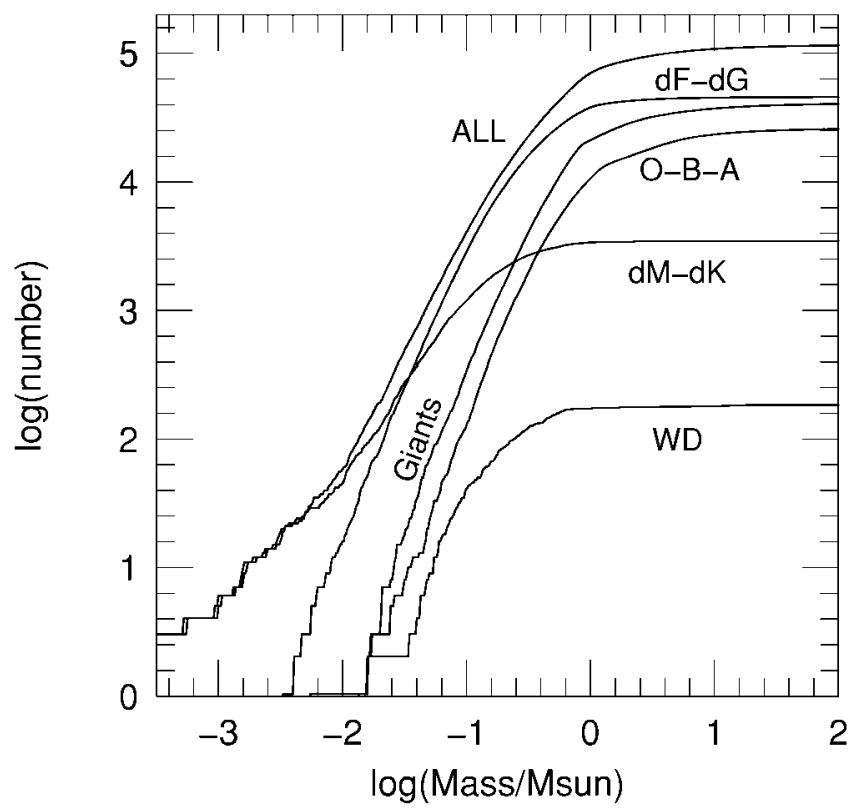

Fig. 2.- Sensitivity of Hipparcos stars to dark binary companions in $P=3.3 \mathrm{yr}$ orbits as a function of companion mass, according to stellar type. Types are broken down (in order of frequency in the catalog) into FG dwarfs, giant stars, O-B-A stars, $\mathrm{K}-\mathrm{M}$ dwarfs, and white dwarfs (WDs). A $5 \sigma$ signal is required for detection. Note that WD, NS, and BH companions of mass $0.6,1.4$, and $7 M_{\odot}$, are respectively detectable among $39 \%$, $68 \%$, and $89 \%$ of all Hipparcos stars. [See the electronic edition of the Journal for a color version of this figure.]

position in the color-magnitude diagram when the parallaxes were sufficiently accurate, and on position in the reduced proper-motion diagram otherwise. In the latter case, distances were assigned based on color, magnitude, and stellar type. The figure shows that white dwarf (WD), $\mathrm{NS}$, and $\mathrm{BH}$ companions of mass $0.6,1.4$, and $7 M_{\odot}$ are respectively detectable among $39 \%, 68 \%$, and $89 \%$ of all Hipparcos stars $\left(N_{\mathrm{Hip}}=118,000\right)$. For periods of $P=1.5 \mathrm{yr}$, these fractions fall to $21 \%, 47 \%$, and $52 \%$. At $P \sim 1$ yr, sensitivity is seriously compromised by parallax aliasing and at shorter periods the sensitivity falls off rapidly. On the other hand, for $P \gtrsim 3.3 \mathrm{yr}$, orbital solutions become rapidly unstable. Hence, the sensitivities peak fairly sharply at $P \sim 3.3 \mathrm{yr}$.

The overwhelming majority of these Hipparcos stars are $\mathrm{F}$ and $\mathrm{G}$ dwarfs, or giant stars whose progenitors are overwhelmingly $\mathrm{F}$ and $\mathrm{G}$ dwarfs. The frequency of companions per $\log$ period for $P \sim 3.3 \mathrm{yr}$ among such stars is $d f_{b} / d \log P \sim 7 \%$ (Duquennoy \& Mayor 1991). From the previous paragraph, Hipparcos is sensitive to companions over about half a dex in period, $\Delta \log P \sim 0.5$. The total number of Hipparcos stars that were born with NS/BH progenitor companions in this period range is then

$$
N_{\text {progen }}=N_{\text {Hip }} \frac{d f_{b}}{d \log P} \Delta \log P f_{\text {progen }}=40 \frac{f_{\text {progen }}}{1 \%},
$$

where we have normalized the fraction of companions that are NS/BH progenitors to $f_{\text {progen }}=1 \%$, in accord with an estimate by Gould (2000) for their relative frequency among all stars (both binary and single). If a fraction $f_{\text {fail }}$ of these progenitors ended their lives as failed $\mathrm{SNe}$, then there should be $\sim 40 f_{\text {fail }} \mathrm{BHs}$ in orbits within the period range cov- ered by Hipparcos, of which Hipparcos should be sensitive to $\sim 80 \%$ of them (from the previous paragraph), giving a total of $\sim 30 f_{\text {fail }}$ BHs. In fact, none of the 235 Hipparcos astrometric binaries with orbital solution (188 have $P<3.3$ yr) contain a clear $\mathrm{BH}$ candidate component. In all cases we find the mass of the companion (if we assume it to be invisible) either well below the $\mathrm{BH}$ range or of the order or smaller than that of the luminous star, which implies that the companion is a main-sequence star that is fainter than the primary.

One would like to use this result to argue that less than $10 \%$ of massive stars end as failed SNe. That is, if more than $10 \%$ failed, we would expect more than three $\mathrm{BH}$ companions. Since we find none, the hypothesis would be ruled out at the $95 \%$ confidence level.

There are, however, two objections to this line of reasoning. First, we do not actually know that at formation the fraction of companions that are NS/BH progenitors is $f_{\text {progen }}=1 \%$. Indeed, there are no observational constraints on this parameter and no theoretical reason to believe (or not to believe) that the fraction of NS/BH progenitors is the same for $\mathrm{G}$ star companions as it is for stars in the field. We address this problem in $\S 3$.

Second, even if this fraction is the same at formation, it could be that the very process of the failed SN disrupts the binary. Certainly, binaries of this sort will very often be disrupted by an ordinary SN. For example, consider a binary composed of an $M=1 M_{\odot}$ and an $m^{\prime}=8 M_{\odot}$ star, the latter of which "instantaneously" ejects $83 \%$ of its mass to become an $m=1.4 M_{\odot}$ NS. Even if the NS receives no kick, the system will become unbound unless it is near apocenter in a fairly eccentric orbit, $e>1-2(m+M) /\left(m^{\prime}+M\right)=$ 0.47 . This eccentricity constraint becomes more severe for larger progenitor masses. Also, many NSs are known to receive a significant kick, often several hundred kilometers per second, which would certainly disrupt the binary. However, in a failed SN, a large fraction of the progenitor would fall back on the BH, so the ratio $(m+M) /\left(m^{\prime}+M\right)$ would be much larger. Hence, at least for the relatively less massive progenitors, the binary would not be disrupted. It remains possible that the $\mathrm{BH}$ remnant would also receive a strong kick in a failed SN, but since the mechanism behind the kick is not well understood, this must remain a matter of speculation.

Finally, we note that for the specific case of the Hipparcos sample, there is some question as to its real sensitivity. ESA (1997, Vol. 3), does not quote a specific threshold of detection, i.e., the required goodness of the fit, but from Figure 3, which shows the distribution of $\alpha / \sigma_{\alpha}$ as a function of period, we judge this threshold to be $\sim 5 \sigma$, i.e., the same value we used in estimating the total number of $\mathrm{BH}$ companions that should have been detected. However, Quist \& Lindegren (2000) simulated the number of detections of $V<7$ MS binaries that Hipparcos should have made (based on a Galactic model and the Duquennoy \& Mayor 1991 binary distribution model) and find that the Hipparcos cata$\log$ should contain between $35 \%$ and $200 \%$ more binaries with $P<3.3 \mathrm{yr}$ orbital solutions (type "O") than it actually does. They suggest that many binaries for which ESA (1997) finds no orbital solution, and thus classifies as type "X," or "G," could have produced an orbit if, for example, the period were known from spectroscopic observations. Careful reanalysis of Hipparcos data might lead to new orbital solutions. 


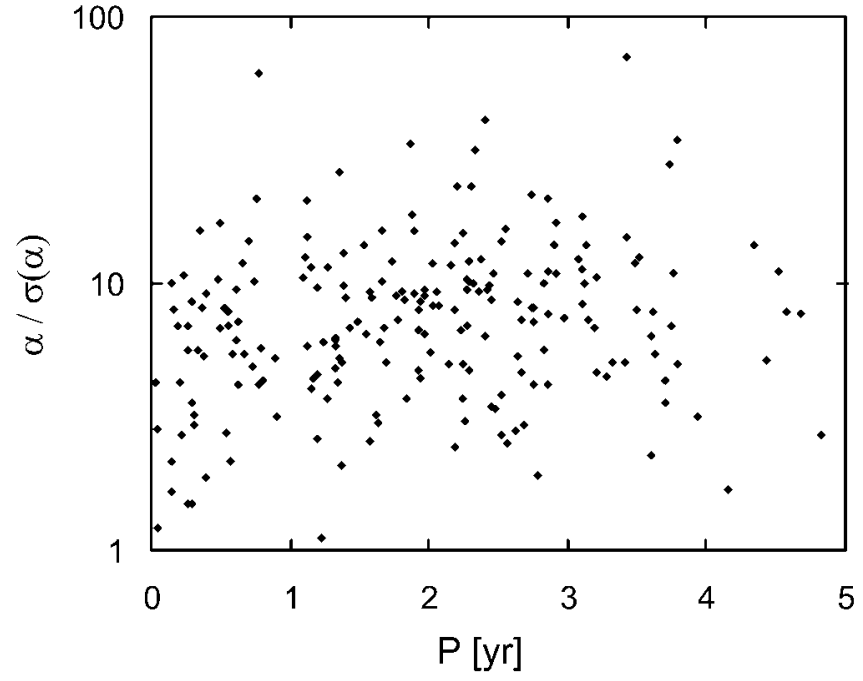

FIG. 3.-Signal-to-noise ratio $\alpha / \sigma_{\alpha}$ as a function of period $P$ for binaries detected in the Hipparcos catalog. From the form of the distribution, we estimate that the catalog is complete down to roughly the $5 \sigma$ detection level.

\section{THE BINARY MASS FUNCTION}

While there is some uncertainty as to the fate of binaries containing failed $\mathrm{SNe}$, the main problem with deriving robust conclusions from equation (2) is that the observational constraints on $f_{\text {progen, }}$ the fraction of binaries born with a massive companion, a NS/BH progenitor, are weak. The main difficulty here is that the "primaries" of these systems (mostly F and G dwarfs and their giant-star descendantssee Fig. 2) are all about $1 M_{\odot}$, whereas the "secondaries" of these systems are substantially more massive. They are therefore both more luminous (making the F-G star difficult to detect) and shorter-lived than the "primaries" (meaning that they are long gone in a field sample of $\mathrm{F}-\mathrm{G}$ stars). Here we present two complementary astrometric methods to overcome this difficulty and show that these can be implemented using future astrometric missions. We will make our specific estimates assuming that the mission parallax errors have the form

$$
\sigma_{\pi}=\left[\sigma_{0}^{2}+\sigma_{15}^{2} 10^{0.4(R-15)}\left(1+c_{\mathrm{RN}} 10^{0.4(R-15)}\right)\right]^{1 / 2},
$$

where the three terms represent the behavior in the systematics-limited, photon-limited, and read-noise-limited regimes. As in the previous section, we will assume that $\sigma_{\alpha}=\sigma_{\pi}$ and that a $5 \sigma$ signal is required for detection. For definiteness, we adopt parameters

$$
\sigma_{0}=38 \mu \mathrm{as}, \quad \sigma_{15}=343 \mu \mathrm{as}, \quad \mathrm{c}_{\mathrm{RN}}=1.063,
$$

which are characteristic of the Full-Sky Astrometric Explorer (FAME; see also Salim, Gould, \& Olling 2002). Finally, we assume a 5 year mission lifetime. At the end of this section, we briefly discuss how our results would be affected if parameters characteristic of other missions were adopted.

We wish to determine the ICMF of G stars as a function of companion mass, $m$, with some period $P$. For definiteness, let us consider a period range $3 \mathrm{yr}<P<5 \mathrm{yr}$ and restrict ourselves to one point of the ICMF: B2 stars corresponding to a mass range $11<m / M_{\odot}<15$ and magnitude range $-2.8<M_{V}<-2.0$. What we seek is the ratio of formation rate of $\mathrm{B} 2-\mathrm{G}$ binaries (in this period range) to the formation rate of all $\mathrm{G}$ stars. From this formulation of the problem, it would seem that one should just survey OB associations and count the number of $B 2-G$ binaries and the total number of $\mathrm{G}$ stars. However, since the IMF of OB associations may be significantly different from the diskaveraged IMF, this procedure would produce a biased result. One must somehow compare formation rates in the disk as a whole.

Assume for the moment that the star formation rate has been uniform over the lifetime of the disk. The ratio we seek, $F_{\mathrm{B} 2 \mid \mathrm{G}}^{P}$, is then given by

$$
F_{\mathrm{B} 2 \mid \mathrm{G}}^{P}=\frac{\Sigma_{\mathrm{B} 2}}{\Sigma_{\mathrm{G}}} \frac{\tau_{\mathrm{G}}}{\tau_{\mathrm{B} 2}} f_{\mathrm{G} \mid \mathrm{B} 2}^{P} .
$$

Here $\Sigma_{\mathrm{B} 2}$ is the column density (number per square parsec) of B2 stars (averaged over spiral-arm and interarm regions), $\Sigma_{\mathrm{G}}$ is the column density of $\mathrm{G}$ stars, $\tau_{\mathrm{G}}$ and $\tau_{\mathrm{B} 2}$ are the lifetimes of $\mathrm{G}$ and B2 stars, respectively (capped by the age of the disk in the case of late $\mathrm{G}$ stars), and $f_{\mathrm{G} \mid \mathrm{B} 2}^{P}$ is the fraction of $\mathrm{B} 2$ stars with $\mathrm{G}$ companions in the appropriate period range $P$. The first two ratios in equation (5) are reasonably well known. The last factor $f_{\mathrm{G} \mid \mathrm{B} 2}^{P}$ is very poorly known but can be measured using $F A M E$, which will probe a volume $\sim 10,000$ larger than Hipparcos did. Then using equation (5), one can calculate from the current fraction of B stars with a $\mathrm{G}$ dwarf companion, the fraction of $\mathrm{G}$ dwarfs that were born with B companions. Figure 4 shows the number of stars for which $F A M E$ is sensitive to dark (or dim, since a

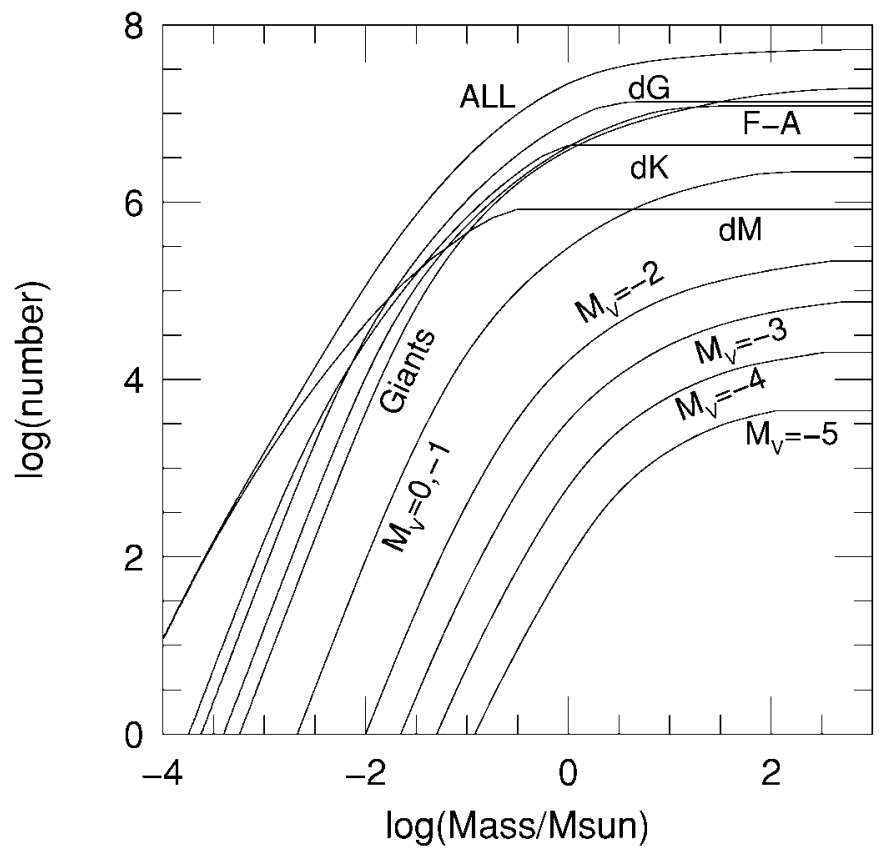

FIG. 4.-FAME sensitivity to binaries with dark (or very dim) companions in $5 \mathrm{yr}$ periods as a function of the dark-companion mass, for various spectral types. The two curves labeled "F-A" and " $M_{V}=0,-1$ " represent WD progenitors, while the four curves labeled " $M_{V}=-2$ " through " $M_{V}=-5$ " represent higher mass main-sequence stars, which are NS or BH progenitors. The calculation follows that of Salim et al. (2002), except that for early-type stars, the disk scale heights are rescaled in accordance with Miller \& Scalo (1979), while the density profile of late-type stars is taken from Zheng et al. (2001). [See the electronic edition of the Journal for a color version of this figure.] 
$\mathrm{G}$ dwarf is much fainter than a B star) companions with a given mass in 5 year orbits, for various spectral types. There are $4 \times 10^{6}$ WD progenitors $\left(1.2 M_{\odot} \lesssim M \lesssim 8 M_{\odot}\right.$, i.e., the $\mathrm{F}-\mathrm{A}$ and $M_{V}=0,-1$ curves in Fig. 4) for which $F A M E$ will be sensitive to companions of $1 M_{\odot}$ (G dwarfs), as well as $2 \times 10^{4} \mathrm{NS}$ or $\mathrm{BH}$ progenitors $\left(8 M_{\odot} \lesssim M \lesssim 20 M_{\odot}\right.$, i.e., the $M_{V} \leq-2$ curves in Fig. 4). If the companion rate in this period range is $7 \% \mathrm{dex}^{-1}$ and $5 \%$ of companions are $\mathrm{G}$ stars, then FAME will detect $\sim 4000 \mathrm{G}$ star companions of WD progenitors within an octave of period and $\sim 20 \mathrm{G}$ star companions of heavier stars. Thus, the WD-progenitor ICMF will be mapped out in great detail and can then be extended with reasonably good confidence into the higher mass range of the progenitors of $\mathrm{SNe}$, luminous or failed. There will also be a direct measurement of the ICMF in this high mass regime, albeit somewhat crude.

Of course, the star formation rate has not been uniform over the lifetime of the disk, but it is straightforward to take account of this variation by modifying equation (5).

This method of determining the ICMF assumes implicitly that $f_{\mathrm{G} \mid \mathrm{B} 2}^{P}$ has not changed from today's value over the age of the disk. While this assumption is plausible, it can also be partially checked using a different application of $F A M E$ astrometry, namely, by detecting the remnant object companions themselves.

In contrast to NS/BH progenitors, the evolution of WDprogenitor binaries is deterministic provided that the pair is not close enough to interact during the asymptotic giant branch (AGB) phase. This is because the mass loss of the progenitor proceeds on timescales that are long compared to the period, so that the evolution is adiabatic. One finds that the final semimajor axis is $a=a^{\prime}\left(M+m^{\prime}\right) /(M+m)$, where $a^{\prime}$ is the initial semimajor axis and $m^{\prime}$ is the initial mass. Hence the initial period $P^{\prime}$ is related to the final period $P$ by

$$
P^{\prime}=P \frac{(M+m)^{2}}{\left(M+m^{\prime}\right)^{2}} .
$$

This means that, while it is not possible to directly probe the time-averaged ICMF in the $\mathrm{NS} / \mathrm{BH}$ progenitor regime (because the binaries could have been disrupted by the $\mathrm{SNe}$ ), it might be possible to probe it in the WD-progenitor regime.

A significant problem in determining the ICMF for $G$ stars is that when a $0.6 M_{\odot}$ companion to a $\mathrm{G}$ dwarf is discovered, one does not immediately know whether this companion is a WD or an M dwarf (with $M_{V} \sim 8$ ). Neither has much luminosity compared to the $\mathrm{G}$ dwarf and so in neither case would the mass determination be significantly affected (see eq. [1]). By the same token, however, there would be no obvious signatures that the companion was one type or the other. It is possible that with precision photometry one could detect the IR excess due to the M dwarf. High signalto-noise ratio spectroscopy could certainly detect the $\mathrm{M}$ dwarf if it were there. The scope of the spectroscopy project would be significantly reduced if one surveyed $\mathrm{K}$ dwarfs rather than $\mathrm{G}$ dwarfs because the $\mathrm{K} / \mathrm{M}$ magnitude difference is substantially smaller than the $\mathrm{G} / \mathrm{M}$ difference. Figure 4 shows that $F A M E$ will be sensitive to WD companions of $10^{6} \mathrm{~K}$ dwarfs and $2 \times 10^{6} \mathrm{G}$ dwarfs, which is certainly enough to obtain a large sample of $\mathrm{WD} / \mathrm{dG}-\mathrm{dK}$ binaries.

The major limitation of this method comes from the fact that during its 5 year mission, $F A M E$ can obtain accurate mass measurements only for $P<5 \mathrm{yr}$. According to equation (6), the periods $P^{\prime}$ of the progenitor systems were substantially shorter than the periods $P$ of their present-day descendants. Specifically, for $\mathrm{G}$ dwarf primaries, we have $P^{\prime}\left(M+m^{\prime}\right)^{2}<12.8 M_{\odot}^{2}$ yr. If the periods were too short, then during the AGB phase, the binary would have suffered mass transfer and its evolution would have taken a complex course. If we assume that no mass transfer occurs for $a^{\prime}>1.5 \mathrm{AU}$ (the exact value is model dependent), then this condition implies $\left(M+m^{\prime}\right)\left(a^{\prime} / \mathrm{AU}\right)<12.8^{2 / 3} M_{\odot}$, or $m^{\prime}<2.6 M_{\odot}$. Hence, only the lower mass WD-progenitor population is probed. Moreover, in this mass range, the WD mass is only a very weak function of the progenitor mass (hence the peakiness of the WD mass function). Given both the astrometric errors and the errors in the photometric masses of the $\mathrm{G}$ star primaries, it seems unlikely that one could obtain much more detail than the total number of WDs as a function of period. Hence, one would really obtain only a single point beyond the usual G dwarf ICMF, which already encompasses secondaries that are fainter and lower mass than the primary. Nevertheless, equal-mass is the only natural scale in this problem. Thus, if this direct determination of the time-averaged ICMF tracked the ICMF measured from present day companions of earlytype stars across the equal-mass boundary, it would lend credence to the latter ICMF measurement at higher masses.

\subsection{Other Missions}

Two other planned astrometric missions are the Deutsches Instrument für Vielkanalphotometrie und Astrometrie (DIVA) and Global Astrometric Interferometer for Astrophysics (GAIA). How well would they be able to detect failed SNe? DIVA is planned to be a 2 year mission whose parallax precisions are given approximately by

$$
\sigma_{0}=158 \mu \mathrm{as}, \quad \sigma_{15}=1380 \mu \mathrm{as}, \quad \mathrm{c}_{\mathrm{RN}}=1.91,
$$

in place of equation (4). Since $D I V A$ is substantially more sensitive than Hipparcos, it would have no difficulty detecting large numbers of binaries with $\mathrm{BH}$ remnants of failed $\mathrm{SNe}$ (if they exist). The question is, could it measure the fraction of $\mathrm{G}$ stars born with WD/NS/BH-progenitor companions? Using equation (7), we find that the number of WD progenitors that can be probed for $1 M_{\odot}$ companions is reduced by an order of magnitude to $5 \times 10^{5}$. This is a significant, but not in itself devastating, reduction. However, the fact that the maximum period probed is reduced from 5 to 2 years means that $D I V A$ in its baseline configuration would not be sensitive to noninteracting systems. This would render difficult the interpretation of the results. However, if $D I V A$ were extended to a 5 year mission, then the number of WD progenitors probed (at $P=5 \mathrm{yr}$ ) would rise to $2 \times 10^{6}$, making it qualitatively comparable to $F A M E$. The number of NS/BH progenitors would then be about 5000 , probably too low to make a reliable measurement.

For $G A I A$, which is a 5 year mission, the corresponding parameters are

$$
\sigma_{0}=2.6 \mu \mathrm{as}, \quad \sigma_{15}=10.2 \mu \mathrm{as}, \quad \mathrm{c}_{\mathrm{RN}}=0.012,
$$

except that in equation (3), $R$ should be replaced by the $G A I A$ astrometric bandpass, which is more similar to $V$ (Perryman 2001). This would permit a relatively modest factor of $\sim 3$ increase in the number of WD progenitors probed 
relative to FAME but would increase the number of NS/ $\mathrm{BH}$ progenitors probed by a factor of 15 .

Additionally, if either FAME or a 5 year version of $D I V A$ is launched during the next few years, and if GAIA is launched a decade hence, then it would be possible to effectively cover periods of $\sim 15$ years for the stars they observed in common.

\section{CONCLUSION}

The notion that some massive stars undergo a collapse without producing a SN first appeared as a failure to produce SN explosions in hydrodynamic simulations, but it later gained ground as a possible mechanism behind GRBs. These theoretical considerations were left without any empirical evidence. If in binary systems these failed SNe col- lapse into BHs without disrupting the binaries, one might be able detect such systems astrometrically. To this end we suggest using space-based missions such as FAME, DIVA, or $G A I A$, since they will observe vast number of stars with great precision. To estimate the rate of $\mathrm{SNe}$ that fail, it is first necessary to estimate the number of binaries that were born consisting of a $\mathrm{G}$ dwarf and a massive, short-lived B star (NS/BH progenitor). We can derive this number using astrometric detections of either currently existing $\mathrm{G}+\mathrm{B}$ pairs, or by extrapolating from the number of $\mathrm{G}+\mathrm{WD}$ binaries. The result of this experiment would either discover a new phenomenon (BH collapsar) or place stringent limits on SN and GRB models.

This work was supported in part by JPL contract 1226901.

\section{REFERENCES}

Beacom, J. F., Boyd, R., \& Mezzacappa, A. 2000, Phys. Rev. Lett., 85, 3568

2001, Phys. Rev. D, 63, 3011

Burrows, A. 1988, ApJ, 334, 891

Duquennoy, A., \& Mayor, M. 1991, ApJ, 248, 485

Gould, A. 2000, ApJ, 535, 928

Gould, A., \& Salim, S. 1999, ApJ, 524, 794

ESA. 1997, The Hipparcos and Tycho Catalogues (ESA SP-1200; Noordwijk: ESA)

MacFadyen, A. I. \& Woosley, S. E. 1999, ApJ, 524, 262

Mikaelian, K. O. 1978, Ap\&SS, 57, 245

Miller, G. E., \& Scalo, J. M. 1979, ApJS, 41, 513

Paczyński, B. 1998, ApJ, 494, L45

Perryman, M. A. C. 2001, A\&A, 369, 339

Quist, C.F., \& Lindegren, L. 2000, A\&A, 361, 770

Salim, S., Gould, A., \& Olling, R. 2002, ApJ, in press

Scalo, J. M. 1986, Fundam. Cosmic Phys., 11, 1

van den Bergh, S., \& Tammann, G. A. 1991, ARA\&A, 29, 363

Woosley, S. E. 1993, ApJ, 405, 273

Woosley, S. E., \& Weaver, T. A. 1986, ARA\&A, 24, 205

Zheng, Z., Flynn, C., Gould, A., \& Salim, S. 2001, ApJ, 555, 393 